15

\title{
Кристалл-дифракционная тестовая установка для измерения заряда нейтрона: испытания тепловой защиты и измерения вибраций
}

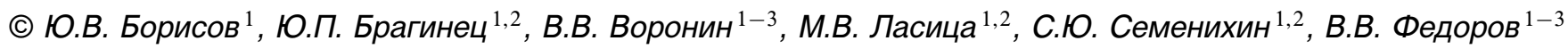 \\ ${ }^{1}$ Петербургский институт ядерной физики им. Б.П. Константинова Национального исследовательского центра \\ „Курчатовский институт“, \\ Гатчина, Ленинградская обл., Россия \\ ${ }^{2}$ Санкт-Петербургский политехнический университет Петра Великого, \\ Санкт-Петербург, Россия \\ ${ }^{3}$ Санкт-Петербургский государственный университет, \\ Санкт-Петербург, Россия \\ E-mail: borisov_yv@pnpi.nrcki.ru
}

Поступило в Редакцию 22 октября 2018 г.

Описана конструкция тестовой установки для ультрапрецизионной нейтронной спектрометрии с использованием дифракции в совершенных кристаллах. Приводятся результаты экспериментов по проверке температурной стабильности внутри установки. Обсуждаются результаты измерений спектра вибраций в рабочей зоне пучка PF1b реактора ILL (Гренобль, Франция), куда предполагается переместить установку для проведения тестовых экспериментов.

DOI: 10.21883/PJTF.2019.02.47226.17571

Современное экспериментальное ограничение на величину заряда нейтрона находится на уровне $\sim 10^{-21}$ заряда электрона [1]. Нами предложен эксперимент [2], прозволяющий в принципе улучшить это ограничение по крайней мере на порядок.

В настоящей работе дается техническое описание тестовой установки. Приводятся результаты экспериментальной проверки температурной стабильности внутри установки, а также результаты измерений спектра вибраций в рабочей зоне пучка PF1b реактора ILL (Гренобль, Франция), куда предполагается переместить установку для проведения тестовых экспериментов.

На рис. 1 показан один из вариантов реализации эксперимента [3], использующий метод спиновой интерферометрии [2] и эффект дифракционной фокусировки $[4,5]$ при дифракции нейтронов на двух совершенных кристаллах в симметричной схеме Лауэ.

Поляризованный вдоль оси $x$ входной нейтронный пучок сначала проходит через магнитную призму $M 1$, которая расщепляет пучок на два с противоположными спинами и направляет на первый кристалл, существенно усиливающий угловое расщепление $(L-$ толщина кристалла, $(h k l)$ - индексы Миллера системы плоскостей отражения, g - вектор обратной решетки, характеризующий эту систему плоскостей). Продифрагировавший отраженный пучок от первого кристалла попадает на второй кристалл с плоскостями отражения, параллельными плоскостям первого кристалла в пределах брэгговской ширины. Отраженный и сфокусированный вторым кристаллом нейтронный пучок после прохождения через призму $M 2$ детектируется. Между кристаллами находится рабочая область 1 . Нейтронный пучок на всем пути от призмы $M 1$ до $M 2$ должен находиться в нулевом магнитном поле $\mathbf{H} \sim$ 0. На рис. 1 область экранирования показана пунктирными линиями.

В такой геометрии дважды отраженный продифрагировавший пучок фокусируется на выходной поверхности второго кристалла [5]. Кроме того, для каждой траектории нейтрона (при отсутствии воздействия на нейтрон в области 1) угол поворота спина в призме $M 1$ равен по величине, но противоположен по знаку углу поворота в призме $M 2$, т. е. суммарный угол поворота спина равен нулю. Расчет магнитных преломляющих призм применительно к рассматриваемой геометрии сделан в [6]. Наличие внешней силы, например электрического поля в рабочей области, приведет при наличии электрического заряда к отклонению направления движения нейтрона на некоторый угол и соответственно к смещению фокуса по выходной поверхности второго кристалла и повороту спина нейтрона [3].

При вполне реальной чувствительности к углу поворота спина нейтрона $\sigma\left(\phi_{e}\right) \sim 10^{-4}$ можно получить ограничение на заряд нейтрона на уровне $\sim 10^{-22}$ от заряда электрона [3], что на порядок лучше современного ограничения. Для изучения эффекта двухкристальной фокусировки нейтронов на раздельных кристаллах была разработана и изготовлена тестовая установка с активной и пассивной тепловой защитой. Установка содержит общее основание (опорную плиту), на котором смонтированы водяные радиаторы для системы термостабилизации, и две поворотные платформы, на которых расположены рабочие кристаллы в кристаллодержателях. Вся установка находится в теплоизолирующем кожухе. Поляризация нейтронов и магнитная система для изучения фокусировки не нужны.

Чтобы обеспечить высокую стабильность угловой юстировки кристаллов и их относительного положения 


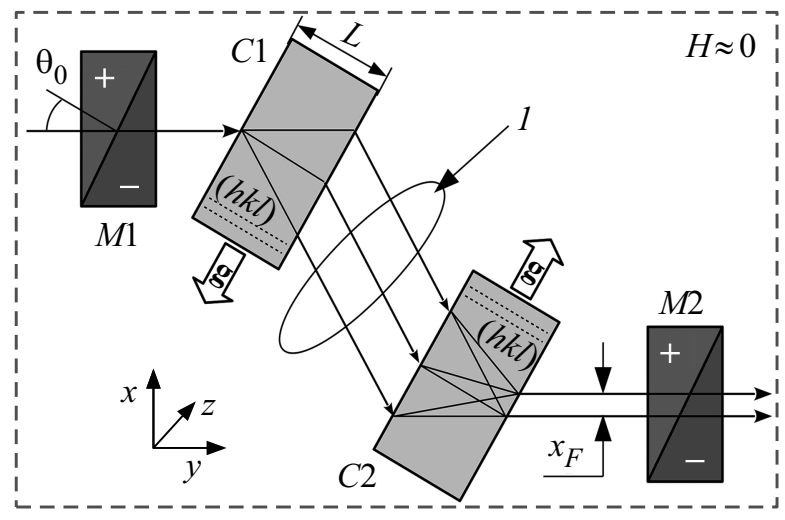

Рис. 1. Схема эксперимента. $M 1, M 2$ - магнитные призмы; области „,+“ и „-““ отвечают противоположным магнитым полям величины $B$; $C 1$ и $C 2$ - первый и второй кристалл соответственно; $\theta_{0}-$ угол между этими областями; $x_{F}-$ смещение пучка при воздействии на нейтроны внешней силы в рабочей области 1 .

во время длительных измерений, в качестве опорной плиты была использована стеклокерамика СО-115М (астроситалл) размером $1000 \times 300 \times 70 \mathrm{~mm} \mathrm{c} \mathrm{коэффи-}$ циентом теплового расширения $<2 \cdot 10^{-8} \mathrm{~K}^{-1}$, изготовленная на заводе оптического стекла (Лыткарино, Московская обл.). В качестве теплоносителя в системе термостабилизации используется деминерализованная вода, протекающая через систему алюминиевых и медных радиаторов, соединенных друг с другом посредством гибких трубок. Алюминиевые радиаторы прикреплены к опорной плите по периметру и на ее нижней поверхности, а медные - вокруг кристаллов. Для обеспечения лучшего теплового контакта между радиаторами и опорной плитой нанесена термопаста (КПТ-8). Организовано встречно-параллельное прохождение теплоносителя через всю систему радиаторов, чтобы уменьшить градиенты температур.

Для прокачки теплоносителя через замкнутую систему радиаторов и поддержания в установке заданной температуры используется циркуляционный пропорционально-интегрально-дифференциальный (ПИД) регулятор Julabo F32-HE (объем бани 81) с возможностью измерения и регулирования температуры с точностью $0.01 \mathrm{~K}$.

Для реализации двухкристальной установки были отобраны два совершенных кристалла кварца размером $100 \times 100 \times 30 \mathrm{~mm}$ с рабочей плоскостью $(100)$ и межплоскостным расстоянием $d=4.25 \AA$. Разброс величины $d$ по всему объему кристаллов составляет $\Delta d / d<10^{-6}$, что на порядок меньше брэгговской ширины отражения.

Установка была смонтирована на испытательном стенде. Проведено ее подключение к циркуляционному регулятору Julabo F32-HE и выполнены гидравлические испытания. Собрана пассивная тепловая защита из экструдированного пенополистирола (пеноплэкса) с внеш- ним слоем из вспененного полиэтилена с отражающей алюминиевой фольгой.

Для стабилизации и контроля температуры и температурных градиентов внутри термостата на ситалловой плите и кварцевых кристаллах было установлено несколько датчиков, представляющих собой платиновые термосопротивления типа Pt-100. Температурные показания с датчиков снимались при помощи цифрового мультиметра Keithley 2700 с возможностью последовательного сканирования всех датчиков при использовании многоканальной платы реле Keithley 7700. На каждом кристалле было установлено по два датчика, позволяющих определить максимальную разность температуры на кристалле. Дополнительно на одном из кристаллов помещался опорный датчик циркуляционного регулятopa Julabo, посредством которого устанавливалась и стабилизировалась рабочая температура.

Целью работы активной тепловой защиты является поддержание постоянной температуры внутри установки независимо от изменений внешних температурных условий. Но из-за наличия массивной опорной плиты из астроситалла с большой теплоемкостью инерционность ПИД-регулятора довольно велика $(\tau \sim 2-3 \mathrm{~h})$, что снижает быстродействие регулятора. Низкое быстродействие приводит к существенному ухудшению стабилизации температуры (из-за запаздывания компенсации регулятором быстрых вариаций температуры). Поэтому был создан второй контур пассивной защиты с конструкцией, включающей воздушный зазор в виде деревянного каркаса, обшитого внутри полиэтиленовой пленкой, а снаружи - слоем из вспененного полиэтилена с отражающей алюминиевой фольгой. Зазор между слоями составлял величину порядка 35-40 mm.

Отрицательное влияние на теплозащиту оказывал поток горячего воздуха от системы охлаждения Julabo, coздававший локальный градиент температуры. Для уменьшения указанного влияния был смонтирован воздуховод для сброса этого воздуха в ствол вентиляционного канала помещения.

При тестовых испытаниях температура вокруг установки искусственно изменялась на несколько градусов, чтобы смоделировать суточные колебания температуры в нейтроноводном зале реактора ILL, где предполагается проводить полномасштабный эксперимент.

Для сравнения температурные измерения проводились как с дополнительным внешним пассивным контуром, так и без него. Измерялись изменения разности температур между двумя кристаллами, расположенными на большом расстоянии $(600 \mathrm{~mm})$, а также изменения градиентов на самих кристаллах (т. е. изменения разности температур между верхней и нижней частями кристалла) в зависимости от разности между температурой окружающей среды и температурой внутри установки, удерживаемой при помощи регулятора Julabo.

На рис. 2 для случая с двойным контуром пассивной защиты показана зависимость разброса разности температур двух кристаллов $(a)$ и разброса разности 


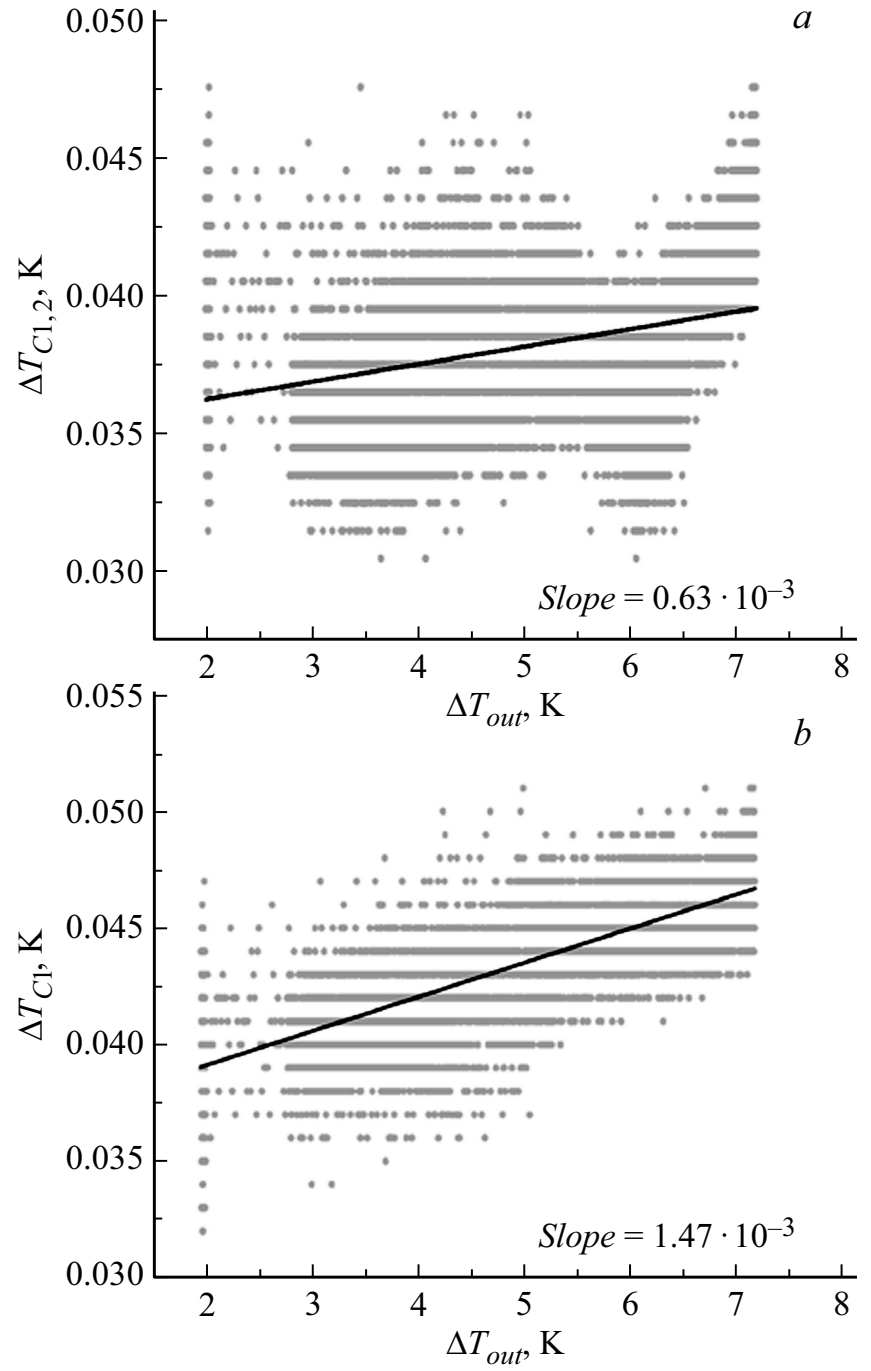

Рис. 2. Зависимость разброса разности температур кристаллов $C 1$ и $C 2(a)$ и разброса разности температур на датчиках одного из кристаллов $C 1(b)$ от разности температур вне и внутри установки $\Delta T_{\text {out }}$.

температур на датчиках одного из кристаллов $(b)$ от разности температур окружающей среды и внутри установки. Точками представлены экспериментальные данные. Сплошная линия - результат усреднения. Измерения показали, что добавление второго контура пассивной защиты дает улучшение стабильности температуры и разности температур кристаллов в несколько раз.

В результате достигнута стабильность температуры на кристаллах не хуже $0.01 \mathrm{~K}$, а разброс температур на кристаллах и разностей температур между ними на уровне $\sim 0.005 \mathrm{~K}$ при колебаниях температуры в помещении до $6 \mathrm{~K}$.

Относительные смещения кристаллов от таких градиентов температур приводят к относительным отклонениям направлений нейтронного пучка, на несколько порядков меньшим брэгговской дифракционной ширины.
В 2017г. на пучке PF1b реактора ILL (Гренобль, Франция) были проведены предварительные измерения уровня вибраций при помощи прибора VA-2 с рабочим диапазоном измерений частот $0.7-1000 \mathrm{~Hz}$. Данный прибор позволяет измерять вибрацию отдельно по трем осям: $H 1$ и $H 2$ - горизонтальные ортогональные оси, $V$ - вертикальная ось.

Основные измерения проводились в режиме измерения скорости. При помощи цифрового осциллографа регистрировался аналоговый сигнал с прибора VA-2. Также проводилось изучение влияния работы насоса циркуляционного термостата Julabo. Результаты измерений представлены на рис. 3.

Предполагается, что основной режим работы насоса во время проведения эксперимента по измерению электрического заряда нейтрона минимальный. Измерения проводились для вертикальной составляющей, поскольку система особенно чувствительна к вибрациям в этом направлении. Из рис. 3 следует, что основной вклад в общий вибрационный фон происходит на частоте $25 \mathrm{~Hz}$ при скорости смещения кристалла $\sim 6.2 \cdot 10^{-4} \mathrm{~cm} / \mathrm{s}$, что дает величину максимальной скорости смещения кристаллов относительно друг друга на уровне $\sim 10^{-3} \mathrm{~cm} / \mathrm{s}$. При этом брэгговская ширина отражения, выраженная в скоростях нейтрона относительно кристалла, составляет $\Delta v_{\mathrm{B}} \sim 1 \mathrm{~cm} / \mathrm{s}$.

Полученные результаты позволяют надеяться на то, что экспериментальная установка для измерения электрического заряда нейтрона кристалл-дифракционным методом с использованием разнесенных на большое расстояние кристаллов даст увеличение современной точности (которая сейчас находится на уровне $\sim 10^{-21}$ от заряда электрона) по крайней мере на порядок.

Работа выполнена в рамках государственного задания Министерства образования и науки РФ 3.3838.2017/4.6.

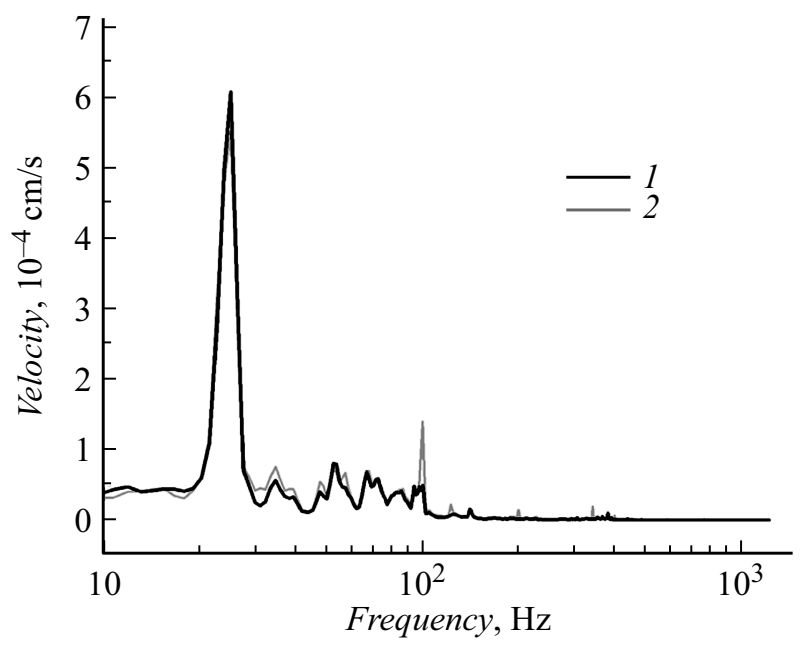

Рис. 3. Измерение уровня вибраций в зависимости от скорости насоса циркуляционного термостата Julabo. 1 - насос выключен, 2 - насос работает на минимальной скорости. 


\section{Список литературы}

[1] Baumann J., Gahler R., Kalus J., Mampe W. // Phys. Rev. D. 1988. V. 37. P. 3107-3112.

[2] Voronin V.V., Akselrod L.A., Zabenkin V.N., Kuznetsov I.A. // Phys. Proc. 2013. V. 42. P. 25-30.

[3] Kuznetsov I.A., Berdnikov Ya.A., Berdnikov A.Ya., Borisov Yu.V., Braginetz Yu.P., Fedorov V.V., Lasitsa M.V., Semenikhin S.Yu., Khorina M.L., Voronin V.V. // J. Phys.: Conf. Ser. 2016. V. 746. P. 1-6.

[4] Инденбом В.Л., Слободецкий И.Ш., Труни К.Г. // ЖЭТФ. 1974. T. 66. B, 3. C. $1110-1120$.

[5] Arthur J., Shull C.G., Zeilinger A. // Phys. Rev. 1985. V. 32. P. 5753-5763.

[6] Борисов Ю.В., Бердников А.Я., Бердников Я.А., Брагинеи, Ю.П., Воронин В.В., Кузнецов И.А., Ласица М.В., Семенихин С.Ю., Федоров В.В. // Письма в ЖТФ. 2015. T. 41. B. 18. C. 1-7. 